\title{
HISTORIA
}

\section{EL EJERCICIO DE LA ENFERMERÍA DOMICILIARIA EN BRASIL Y EN EL MUNDO. UNA VISIÓN HISTÓRICA Y ÉTICA}

Taka Oguisso

Licenciada en enfermería y abogada. Doctora en Salud Pública y Profesora Titular de la Escuela de Enfermería de la Universidad de San Pablo. Brasil.

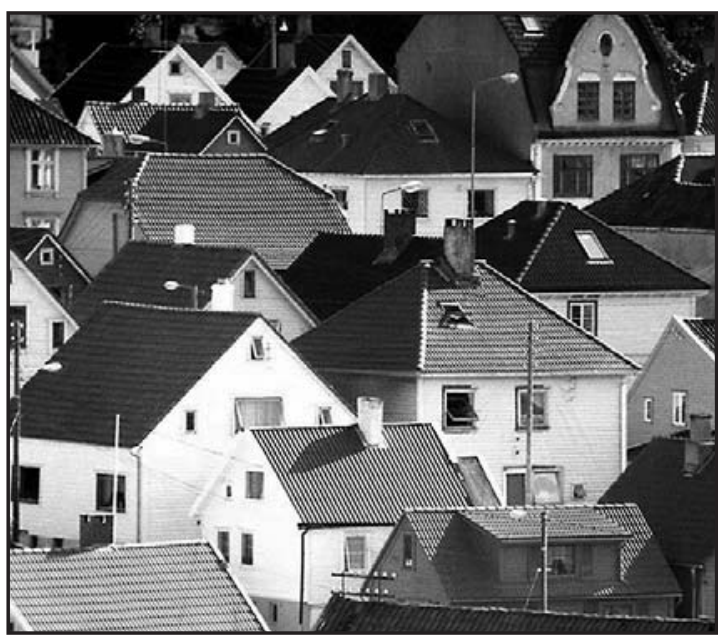

NURSING HOME CARE IN BRASIL AND THE WORLD. A HISTORICAL AND ETHICAL VIEW

\section{SUMMARY}

7 he origins of home care are described, as well as its definitions and objectives according to the World Health Oganisation, and the way it is developed in some countries in which the system has been implemented with a new modality involving less professional training, by means of carers or comforters, in order to ensure people's comfort, especially that of the elderly at home. Home nursing care delivered in Brasil by nurses employed for this purpose is described as an example, as well as the ethical and legal issues that may arise. In European countries there are certain specific requirements such as previous hospital experience and specialised training in public health and/or obstetric nursing, for nurses to deliver home care, which is understandable considering the legal and technical responsibilities for the nurse who will be alone in the home with no one else to discuss decisions with.

Key words: nursing, history of nursing, home care.

O EXERCÍCIO DA ENFERMAGEM DOMICILIÁRIA NO BRASIL E NO MUNDO: UMA VISÃO HISTÓRICA E ÉTICA

\section{RESUMO}

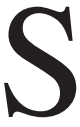
ão descritas as origens da atenção domiciliária, suas definições e objetivos de acordo com a Organização Mundial de Saúde e como esta modalidade de atenção está sendo desenvolvida em alguns países que têm implantado o sistema, inclusive uma modalidade nova e com menor preparo profissional, como cuidadores ou confortadores, para atender o conforto das pessoas, em especial de pessoas mais idosas em suas próprias casas. Como exemplo da prática de enfermagem domiciliar apresenta-se como tal modelo é aplicado no Brasil, com enfermeiras empresárias voltadas para esta atenção, e os problemas éticos e legais que podem advir. Nos países europeus há certas exigências específicas como experiência profissional prévia no hospital e formação especializada em saúde pública e/ou enfermagem obstétrica, para que os enfermeiros possam desenvolver seu trabalho em home care, o que é compreensível, considerando as responsabilidades técnicas e 
legais que irão assumir, pois o profissional estará sozinho no domicílio do paciente, não tendo com quem discutir a melhor decisão a ser tomada.

Palavras-chave: enfermagem, história da enfermagem, atenção domiciliar.

\section{RESUMEN}

e describe los orígenes de la atención domiciliaria, sus definiciones y objetivos de acuerdo con la Organización Mundial de la Salud y cómo esta desarrollado en algunos países que han implantado el sistema hasta una modalidad más nueva y con menor preparación profesional, como cuidadores o confortadores, para atender el confort de las personas, en especial las de más edad en sus propias casas. Como ejemplo de la práctica de enfermería domiciliaria se presenta como es aplicado en Brasil, con enfermeras empresarias para esta atención, y los problemas éticos y legales que pueden ocurrir. En los países europeos hay ciertas exigencias específicas como experiencia profesional previa en hospital y formación especializada en salud pública y/o enfermería obstétrica, para que los enfermeros puedan desenvolver su trabajo en home care, lo que es comprensible considerando las responsabilidades técnicas y legales que irán asumir, pues el profesional estará sólo en el domicilio del paciente, no teniendo con quien discutir la mejor decisión a tomar.

Palabras clave: enfermería, historia de la enfermería, atención domiciliar.

\section{INTRODUCCIÓN}

En los orígenes de la humanidad, en los tiempos del hombre pre - histórico, los nómadas y la incipiente sociedad de entonces vagaba por las selvas en la búsqueda de caza, pesca y colecta de frutos silvestres. Esa instintiva aventura de supervivencia era, seguramente, acompañada de numerosos riesgos de muerte y de heridas, principalmente cuando se trataba de enfrentar animales en busca de alimentos.

Cuando acontecía un accidente o enfermedad, el instinto gregario y la propia vida nómada lo obli- gaban a recibir los posibles cuidados en el propio local del evento o en el abrigo de su casa. Históricamente, pues, se puede decir que los cuidados dispensados a una persona en su propia habitación es una de las más antiguas - aún rudimentarias - formas de atención al ser humano. Sin duda, eran los orígenes de aquello que, hoy, millones de años después, en la terminología básica en salud, seria llamada de "enfermería domiciliaria o home care".

La verdad, "los cuidados existían desde que surgió la vida, una vez que los seres humanos como todos los seres vivos - siempre precisaran de cuidados". "Cuidar es el acto de vida que tiene como fin, primero y antes que todo, permitir que la vida continúe desarrollándose y así luchar contra la muerte: muerte del individuo, muerte del grupo, muerte de la especie"(Collière).

En la trayectoria evolutiva de la sociedad, la acción de cuidar o de hacerse cargo de personas, en el sentido de ayudarlas a cuidar de sí y de atender sus necesidades vitales, se confunde en el tiempo con el trabajo de la madre que nutre sus hijos y de ellos cuida, así como cuida de otras personas dependientes, como los ancianos, heridos y enfermos. La protección materna instintiva fue la primera manifestación de cuidados del ser humano con sus semejantes.

El tiempo pasó y, en la medida que aquellos grupos humanos abandonaban el nomadismo se fijaban en determinado territorio, formando los primeros asentamientos humanos, surgiendo organizaciones primitivas sociales donde hombres $\mathrm{y}$ mujeres asumían funciones distintas en ese embrión de la sociedad, los hombres dedicándose a la caza y a la pesca, con todos los riesgos inherentes a esas tareas, y las mujeres más dedicadas para los trabajos domésticos. Ambos, por lo tanto, dedicados a cuidar "mantener la vida de los seres humanos con el objetivo de permitir la reproducción y la perpetuidad de la vida del grupo" Carvalho, 1965 (Collière).

Con la evolución de las ciencias y el progreso de la tecnología, la asistencia a la salud comenzó a ser transferida de las casas para las instituciones hospitalarias, consideradas el ambiente más seguro y apropiado para la prestación de los servicios que ellos necesiten. Mas con la implantación de recur- 
sos cada vez mayores y más sofisticados, la asistencia hospitalaria se fue tornando cada vez más cara y menos accesible a la población, especialmente a la rural.

El servicio de enfermería domiciliar, iniciado sin pretensión y de forma modesta, en el siglo XIX en Brasil, se transformó en una gran realidad mundial impulsando la asistencia a la salud y a la propia enfermería para nuevos rumbos y desafíos y abriendo caminos para profesionales emprendedores. Esa asistencia de enfermería domiciliar o prestada fuera del ambiente institucional, hospitalario es también conocida como home care.

Son objetivos de este estudio discutir el papel del enfermero en esta modalidad de atención de enfermería prestada fuera del contexto institucional de salud y los beneficios para con el paciente y su familia.

\section{DEFINICIONES Y OBJETIVOS DE HOME CARE}

Esa asistencia es definida por la Organización Mundial de la Salud como aquel cuidado a la salud proporcionado a las personas en su domicilio (OMS, 1999). Ese cuidado debe ser apropiado, de alta calidad y con la relación costo-beneficio compatible para los individuos, que deben mantener su independencia y la mejor calidad de vida posible. Estudios de la Organización Mundial de la Salud demuestran que el home care esta virtualmente asociado con mejor calidad de vida y que la relación costo-beneficio es más frecuentemente favorable en los casos que sustituirían largas permanencias (WHO, 1999).

Son objetivos de home care: sustituir hospitalización repentina por necesidad aguda de cuidado, sustituir una larga internación institucional; prevenir la necesidad de internación institucional y mantener los individuos en su propio domicilio $\mathrm{y}$ comunidad.

A pesar de que los cuidados de home care puedan ser prestados directamente por profesionales de la salud con escolaridad superior, como médicos y enfermeras, la literatura apunta más a los cuidadores formales, de escolaridad media, o informales preparados, especialmente en los países que pretenden reducir costos, dar comodidad al paciente y prevenir posibles infecciones hospitalarias.
De cualquier forma la tendencia mundial es de que home care, como cuidado requerido por el usuario en el domicilio, continuará creciendo, así como el nivel, el volumen y la intensidad y hasta la complejidad de ese cuidado, pues es posible transferir el paciente de una unidad de terapia intensiva para su domicilio o atender la demanda de pacientes crónicos, inclusive reduciendo "la exposición al riesgo de infección hospitalaria, evitando la pérdida de la convivencia familiar e ingresos hospitalarios, además de la disminución de su costo (Dal Bem, 2005).

De hecho, en la discusión sobre la salud en el mundo, es necesario considerar algunos asuntos básicos como la irreversibilidad de la globalización económica, los cambios demográficos y epidemiológicos en los próximos veinte años que, por ejemplo, en Brasil habrá un aumento de más de $200 \%$ de la población anciana. De manera paradójica es el mundo desarrollado o llamado Primer Mundo, se volvió rico antes de llegar a anciano, al paso que en los países sub-desarrollados, las personas se volvieran ancianas antes de ser ricas. Otro asunto a considerar es respecto a la dependencia del paciente y el trabajo de prestación de cuidados, llevando siempre en cuenta las actividades de la vida diaria (AVD) y la utilización de la alta tecnología.

Aparentemente es muy simple satisfacer esas necesidades, como alimentación, eliminaciones, hidratación, higiene, respiración, etc. el cuidador no imagina cuantas actividades envuelven las AVD, pues un simple baño puede necesitar de muchos cuidados cada día, durante los siete días de la semana y en los 365 días del año. El suceso de ese cuidado está apenas en el estar próximo, o al lado del paciente, para alcanzar las cosas que él necesita. La hidratación puede ser necesaria tanto de día como de noche y así por delante (Hirschfeld, Oguisso, 2002).

Al desenvolver una AVD, no siempre puede percibirse cuan ardua puede ser la tarea de dar un baño, desvestirlos para ir al baño o al uso del sanitario. Para algunos pueblos y culturas puede ser una situación extremamente vergonzosa y complicada tanto para los padres como para los hijos, que no fueron preparados y ni acostumbrados a prestar ese tipo de cuidados para los progenitores. En fin, la situación práctica puede envolver muchos pro- 
blemas de difícil solución, dependiendo de la cultura, costumbres y valores sociales.

Además de esas actividades personales habrá otras necesidades como compras en supermercado, uso de transporte público, el control de las propias cuentas financieras, en fin el control de varios medicamentos, que precisan ser administrados en sus dosis, vías y horarios correctos, que en un hospital es un procedimiento simple, pero en home care puede ser complicado y confuso para el cuidador o para el propio paciente. Por otra parte, el paciente necesita de comunicación, apoyo emocional, observación de su comportamiento, administración de los cuidados relacionados a los síntomas, a la dieta, ejercicios físicos, reposo, medicamentos, cuidados con la piel, etc. Sí en el hospital es fácil, para un cuidador (LIDAR convivir) con situaciones como dolor agudo, desmayos, tos prolongada es muy difícil.

Casi todo lo que se realiza en una Unidad de Terapia Intensiva, hoy puede hacerse en el domicilio, debido a la alta tecnología en home care, tales como la nutrición artificial, hidratación, ventilación mecánica, aspiración continua de secreciones, infusiones endovenosas y diálisis que transforman las residencias en verdaderos servicios de enfermería de hospital.

\section{FACTORES A CONSIDERAR EN HOME CARE}

En el desarrollo de la asistencia en home care hay algunos aspectos importantes a tener en cuenta (Hirschfeld, y Oguisso, 2002).

1. La naturaleza del programa considerando la población - objetivo, el tipo de asistencia de 24 horas y en todos los días de la semana.

2. Los criterios para la elección de los pacientes deben incluir la edad, el tipo de enfermedad, el grado de dependencia, la relación con el cuidador, el grado de apoyo familiar y las condiciones económicas para asumir financieramente el costo.

3. La evaluación de los criterios de elección debe ser en relación al nivel y al tipo de cuidado necesario.

4. Los beneficios que el paciente y sus familiares pueden obtener con la opción de home care, sea con la prestación de cuidados de enfermería en las diversas modalidades de cuidado-día o otros específicos, como orientación, consejos, provisión de material y/o medicamentos prescritos, entre otros beneficios.

5. La ejecución del programa de home care puede ser a nivel gubernamental o administrado en conjunto con otras organizaciones, inclusive del sector privado, contemplando la planificación de la asistencia a ser prestada, la educación o preparación de los funcionarios o trabajadores y el control de garantía de calidad.

6. El financiamiento de los servicios de home care puede ser obtenido por medio de impuestos o un sistema de seguro de providencia social. Excepto esas posibilidades, el usuario tendrá que solventar con los costos de su propio bolsillo.

7. La cobertura de los servicios incluye la necesidad de estimar la parcela de la población que precisará de home care, la localización de estos servicios, abarcando tanto el área urbana como el área rural, las categorías de las enfermedades, la edad de la población-objetivo y los niveles de dependencia o incapacidad de los posibles pacientes.

8. El cálculo del costo implica el costo total, el costo-hora, la proporción de trabajadores remunerados o voluntarios, el costo de educación, de los medicamentos, material y del transporte.

\section{HOME CARE EN OTROS PAÍSES}

En países de Europa, como el Reino Unido, Irlanda, Alemania y Suiza así como en los Estados Unidos y Canadá, las altas hospitalarias son cada vez más precoces y el seguimiento es hecho por enfermeros. En los países escandinavos, por tener ya una larga tradición de asistencia domiciliar, el aspecto económico es considerado secundario, colocándose el aspecto ético preferente y la comodidad del paciente de quedar en su propia casa, como el argumento principal y más importante para desenvolver programas de asistencia residencial. Ya en los países de Europa Central, como Hungría, o República Checa y el Este de Europa, como Slovenia el ímpetu para 


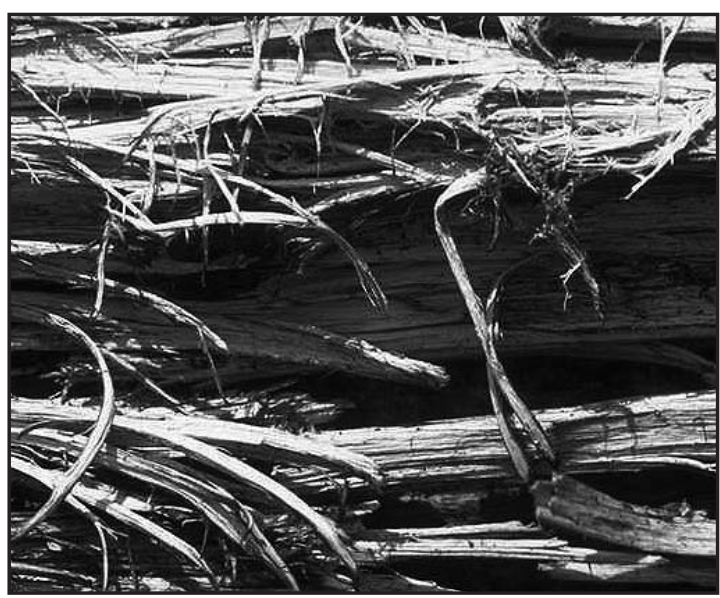

abrazar la atención residencial es esencialmente económico.

En los países con PBI medio, como Corea del Sur, los servicios no tienen cobertura universal, y hay una mezcla entre servicios públicos y privados. En los países de PBI más bajo, pueden existir servicios de cobertura universal como ocurre con Cuba e iniciativas de home care basados en las comunidades locales como en África del Sur, Uganda, Zambia, Quenia, Caaambodia, Tailandia y China (Hirschfeld MJ y Oguisso T, 2002).

La actual tendencia es para la creación o expansión de servicios residenciales, la asistencia a pacientes crónicos, y agudos, desde el pre-operatorio al post-operatorio, perinatal, cuidados paliativos y hasta eventos episódicos. Podría ofrecerse también cuidado-día o cuidado-noche para los ancianos crónicos, cuidado-alivio para pacientes terminales, cuidado-niño enfermo, cuidado-día quirúrgico, consejos sobre los cuidados de todos los tipos y hasta mismo servicios de salud ocupacional y salud escolar para empresas. Otra tendencia que comienza a ser practicada es el programa de preparación específica de LEIGOS, son cuidadores, para ancianos, enfermos crónicos o niños con alguna enfermedad prolongada en domicilio.

Recientemente fue creado un servicio de "comfort keepers"con personas para dar, asegurar o mantener la comodidad, una especie de confortadoras profesionales. Es un servicio creado en 1997, en los Estados Unidos, por la enfermera Kris Clum, que trabajaba en home care. Era inicialmente un cuidado simple y diario, como calentar la leche u ofrecer una prosa amigable, más que se volvió una solución para dar comodidad y ayudar a las personas ancianas para que continúen en sus propias casas con el más alto nivel posible de independencia y autonomía. La asistencia de confortador provee compañía, preparación de comida, ayuda para las deambulaciones diarias o para el traslado del paciente para la consulta médica o exámenes de laboratorio, agencias bancarias, iglesias, pequeñas compras y arreglos o limpieza de la casa. Puede también auxiliar en la higiene, ducha y cuidados en situaciones de incontinencia. Confortadoras pueden susstituir a los cuidadores familiares que cuidan regularmente de esas personas dándoles posibilidad de descansar o dar día libre una que otra vez para cuidar de sus propios intereses, o para dar alguna comodidad que el familiar prefiera no hacer, por ejemplo, cuando un hijo cuida de su madre anciana ésta puede sentir vergüenza en recibir ayuda de él para su higiene personal.

En Alemania, Dinamarca e Israel fueron aprobados reglamentos y criterios para la prestación de atención residencial de acuerdo con determinados patrones de la práctica médica y de enfermería, con metodologías profesionales apropiadas, documentación del tratamiento realizado y control externo de calidad. Segun Ehrenfeld, 1997, son necesarias, cuatro condiciones para selección de pacientes que deseen recibir asistencia domiciliaria:

1. Diagnóstico del paciente, que permita elaborar plano de asistencia de enfermería.

2. Condición estable del paciente que no requiera supervisión constante.

3. Existencia de un lugar con cuarto, cama, baño y otras facilidades mínimas.

4. Existencia de una persona que quiera asumir la responsabilidad por el paciente, o sea un cuidador.

En Suiza (Fondations des services d'aide at de soins à diomicile, 2004), home care comenzó, verdaderamente, en 1920 cuando la Cruz Roja instaló un dispensario de higiene social y creo un grupo de enfermeras visitadoras. El trabajo que ellas desarrollaban estaba relacionado con problemas de alcoholismo, tuberculosis, enfermedades venéreas, prestaban cuidados a recién nacidos, la educación de las madres, distribución de alimentos y car- 
bón a las casas. Actualmente el servicio de home care es definido como aquellos prestados a las personas cuyo estado de salud física o mental exige cuidados controles específicos o ayuda temporal o prolongada. Se trata de un servicio básicamente prestado por enfermeras con experiencia en el campo hospitalario y que llevan al domicilio la asistencia materno-infantil además de los cuidados de higiene y tratamientos bajo prescripción médica, tales como curaciones, administración de medicamentos, suero, transfusión de sangre, alimentación parenteral, quimioterapia, control de glucemia, y cuidados paliativos. Los pacientes atendidos por esos servicios pueden recibir también auxilio en ciertos trabajos domésticos como en la preparación de la comida, acompañamiento en actividades externas (consulta médica, compras, etc.) o servicios de tele-alarma (la persona usa una pulsera especial que puede accionar un sistema de atención de emergencia), entrega de comida preparada o ingreso domiciliario, cuando las condiciones del paciente permiten. Hay también casas apropiadas para esas personas con estructura para una vida semi-independiente o totalmente dependiente. El sistema incluye también el SOS Farmacéuticos, que atiende llamadas de enfermeras para suplir necesidad de medicamentos.

Madden (1997) refiere que en Irlanda, home care es representado por un amplio espectro de servicios prestados por enfermeros graduados a nivel universitario. Sus requisitos son experiencia mínima de 3 años de ejercicio profesional en hospital, debiendo tener aún formación en salud pública y especialización en enfermería obstétrica, para trabajar en home care. Esas exigencias son justificadas porque se presume que el enfermero en trabajo en el domicilio deba ser capaz y competente para tomar decisiones relativas a la promoción, prestación de la salud principalmente en la prestación de los cuidados necesarios a la asistencia del paciente.

Hirschfeld y Oguisso (2002) refieren que home care implica que "en el día a día es preciso tomar decisiones y resolver problemas con el paciente, o en su nombre, en relación a intervenciones médicas, ingreso en el hospital, rutinas diarias y control del dolor y otros síntomas, además de decidir sobre el lugar donde la persona debe vivir y morir". En esa circunstancia es necesario analizar los riesgos, garantías, gastos, protección contra eventuales descuidos o malos tratos, procurando equilibrar los recursos existentes, las preferencias y capacidades tanto del paciente como del cuidador.

En Noruega, Davoy (1997) refiere que home care fue implantado en 1972 y desde 1984 fue aprobada una legislación en que todos los municipios son obligados a proveer a los pacientes de cualquier edad una asistencia a las necesidades de salud, físicas o mentales, que incluye tratamiento y cuidados de enfermería en sus propias casas. Para integrar la mayor participación posible de los propios pacientes y familiares en las actividades asistenciales los enfermeros les ofrecen todo tipo de información, apoyo y orientación sobre los cuidados necesarios. Los costos crecientes de la asistencia hospitalaria hicieron que los pacientes recibiesen alta cada vez más precoz, aumentando significativamente las necesidades de los pacientes en relación al seguimiento clínico, tratamiento, rehabilitación y cuidados especializados de enfermería. Un reglamento gubernamental de 1993 estipuló que para la manutención de la calidad de asistencia domiciliar, la lideranza debería ser ejercida por un enfermero habilitado. Es necesario recordar las condiciones geográficas y climáticas de Noruega para entender que muchas veces el aislamiento del profesional en áreas inhóspitas por largos meses exige de él conocimiento, habilidades y capacidad para tomar decisiones en situaciones extremamente difíciles. De ahí la exigencia de que el enfermero en home care sea un profesional con experiencia en la práctica de enfermería.

Constituye un conjunto único el periodo total de internación institucional, el seguimiento ambulatorio, cuando necesario y los cuidados a ser prestados en el domicilio. Así, las actividades de enfermería comunitaria y domiciliaria son realizadas en estrecha colaboración con todos los servicios de salud, hospitales, casas de reposo o ambulatorios, y muchos de esos servicios se vuelven ininterrumpidos funcionando 24 horas por día, con la atención de llamadas telefónicas. La intención es sustituir, las clínicas de reposo por home care. Enfermeros de salud comunitaria y domiciliar están comenzando también a trabajar estrechamente con organizaciones de voluntarios, recibiendo subsidios guber- 
namentales y cooperación en los cuidados, especialmente de ancianos o personas con deficiencia física (Davoy, 1997). En Hungría la búsqueda de alternativas en la prestación de asistencia a la salud encontró en home care la solución, (Mucha, 1997). Los enfermeros comenzaron a familiarizarse con los estudios para implantación, en 1994, necesitaban prepararse adecuadamente para asumir nuevas responsabilidades con cursos de 40 horas, pero se consideraron insuficientes. Para sanar esa dificultad fue desarrollado un programa de mentores en salud de la familia para ayudar a enfermeros que fuesen a actuar en home care, inicialmente eran médicos la mayoría de los mentores, mas ya existen también enfermeros. Enfermeros Húngaros, conscientes de la necesidad de mejorar la preparación presentaron un proyecto para la creación del curso de nivel universitario para formación de enfermeros en salud pública o salud comunitaria para desarrollar el tipo de servicio prestado a los pacientes en domicilio y enfatizar la asistencia primaria de salud como base para la prestación de la asistencia domiciliar como parte de la propia reforma del sistema de salud húngaro.

Ese énfasis para los cuidados primarios de salud, también es una tendencia en los países europeos no significando abandono de las especializaciones y de la tecnología, mas los gobiernos deben definir criterios y prioridades y crear los servicios necesarios con el debido equilibrio. Es necesario recordar que la hospitalización es apenas un episodio transitorio en la vida de las personas. Pero los enfermeros debemos concentrar esfuerzos y estar preparados para los varios niveles de prevención y promoción de la salud y no es apenas para tratar y cuidar de pacientes institucionalizados.

\section{HOME CARE EN EL BRASIL}

Actualmente existen centenas de empresas brasileñas especializadas en atención de salud domiciliar, la mayoría en el Estado de San Paulo, que atienden más de 30 mil pacientes por mes. La mayoría de los pacientes $(73 \%)$ es constituida por clientes de planos de salud y apenas $20 \%$ pertenecen a la red pública del Sistema Único de Salud (SUS). Inicialmente fue el servicio público que se interesó por esa modalidad en la década de 1960 y el sector privado comenzó al final de la década de
1980. La atención al domicilio pasó a ser incentivado por los propios planos de salud no apenas por la calidad y confort para el paciente, sino por el menor costo (cerca de $30 \%$ en media) que el ingreso hospitalario (Lopes, 2006).

La Agencia Nacional de Vigilancia Sanitaria ANVISA - del Ministerio de Salud del Brasil, bajo la Resolución $\mathrm{n}^{\circ}$. 11, de 26 de enero de 2006 (Brasil. Ministério da Saúde, 2006), dispuso sobre el reglamento técnico de funcionamiento de servicios públicos o privados que prestan atención domiciliaria, sean acciones de promoción a la salud, prevención, tratamiento de enfermedades y rehabilitación desarrolladas en domicilio. La asistencia domiciliaria es entendida como el conjunto de actividades de carácter ambulatorio, programadas y continuadas, desarrolladas en domicilio, el ingreso domiciliario constituye un conjunto de actividades prestadas en el domicilio caracterizadas por la atención en tiempo integral al paciente con cuadro clínico más complejo y con necesidad de tecnología especializada. Ese reglamento reconoce el cuidador como la persona con o sin vínculo familiar capacitada para auxiliar al paciente en sus actividades de la vida cotidiana.

El paciente admitido para recibir atención domiciliario, debe contar con un Plano de Atención Domiciliar o PAD, que consiste en un documento que contiene las medidas que orientan la actuación de todos los profesionales envueltos en la asistencia, desde la admisión hasta el alta. Esos profesionales constituyen el Equipo Multiprofesional de Atención Domiciliar - ENAD - con la función de prestar asistencia clínico-terapéutica y psicosocial al paciente en su domicilio. Finalmente la empresa o institución, pública o privada responsable por la gerencia y operacionalización de la asistencia o internación domiciliar es llamada de SAD, o servicio de atención domiciliar (Brasil. Decreto n. 94.406, de 8 de junho de 1987).

Esta resolución destaca la cuestión del registro en la historia clínica domiciliaria especificando la prescripción de la asistencia clínico-terapéutica y psicosocial para el paciente con previsión para la periodicidad de informes de evolución y acompañamiento y la necesaria revisión del PAD por el profesional de salud que acompaña el paciente y por el responsable técnico del SAD. La historia clí- 


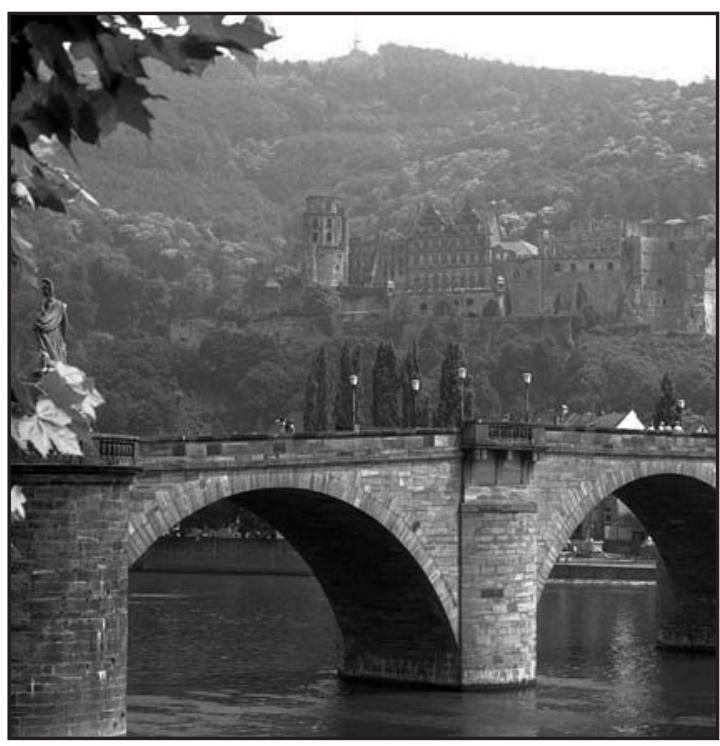

nica domiciliar debe contener el registro de todas las actividades realizadas en la atención directa al paciente, así como la prescripción y evolución multiprofesional, resultados de exámenes, descripción del flujo de atención de urgencia y emergencia, teléfonos de contacto del SAD y orientaciones para llamados.

Otro punto a destacar en la Resolución es el de haber retirado de la palabra prescripción la posibilidad de ser o parecer un término privado de la práctica médica, con eso se admite que otros profesionales de la salud prescriban, tales como psicólogos, enfermeros y fisioterapeutas. Hubo, pues, una evolución en la aceptación del término prescripción para otros profesionales y no apenas para el médico. Dal Ben (2005) refiere al inicio de su emprendimiento en home care, en 1991, "Los administradores y directores de empresas de planos de salud representadas en general, por profesionales médicos, no entendían y repudiaban los términos de consulta y prescripción de enfermería afirmando que eran palabras privativas de la práctica médica". Por esa razón, esa enfermería tuvo que sustituir los términos de consulta de enfermería y prescripción de enfermería por evaluación de enfermería y cuidados de enfermería, respectivamente.

Según esa autora (Dal Bem, 2005) el papel del enfermero en home care está basado en la consulta de enfermería que envuelve la evaluación del esta- do clínico y psicosocial del paciente, inmerso en su realidad domiciliar, de la cual emerge la planificación de la asistencia. Para realizar el ingreso domiciliario es preciso conocer la estructura del ambiente que recibirá el paciente, la dinámica familiar envuelta, la previsión y provisión de material, medicamentos y equipamientos necesarios para cuidar bien y como adecuar el perfil de los profesionales que trabajarán en el espacio del domicilio del paciente. El ambiente hospitalario es, por así decir, la casa o espacio de trabajo de los profesionales de la salud y en cuanto los pacientes serian los huéspedes temporales, son ellos los que tocan el timbre para llamar al profesional, y en el domicilio del paciente él está en su propia casa, y los profesionales necesitan tocar el timbre para que sean admitidos en la casa donde estarán temporalmente.

En los países europeos donde ese sistema de asistencia domiciliar fue implantado hace mucho tiempo, los enfermeros actúan con gran autonomía e independencia desarrollando sus actividades en la asistencia directa, en la realización de procedimientos de enfermería y en la educación para la salud como una extensión de la asistencia hospitalaria. En Suiza (Fondations des services d'aide at de soins à diomicile, 2004), la enfermera de ligación (infirmière de liaison) que coordina la planificación de los cuidados en domicilio, hace la ligación o crea la continuidad entre el hospital y el domicilio. Por eso, esa planificación comienza antes del alta hospitalaria del paciente, identificando sus necesidades y de su familia, promoviendo encuentros y reuniones de los profesionales del equipo, para que los objetivos de la atención y la necesidad del paciente sean atendidos de la forma más racional posible.

Básicamente la calidad de la asistencia domiciliar debe ser un reflejo del nivel de satisfacción del cliente (paciente y familia) y de los miembros del equipo, pues la mayoría de las quejas de insatisfacción siempre envuelven deficiencias en la atención de enfermería. Por eso, el papel del enfermero es fundamental, basado en la consulta de enfermería que implica la evaluación del estado clínico y psicosocial del paciente, inmerso en su realidad domiciliar de la cual emerge la planificación de la asistencia. Esta autora refiere que en caso de inter- 
nación domiciliar es necesario conocer la estructura del ambiente donde el paciente será acogido así como la dinámica familiar, para que de esa forma se adecue el perfil de los profesionales que han de ser seleccionados para atender al paciente.

En el ambiente hospitalario en situación de muerte el médico constatará el óbito y firmará el certificado, la enfermera cuidará de la higiene y taponamientos del cuerpo para prevenir el reflujo de secreciones. En circunstancias normales la rutina del hospital determina que el cuerpo sea trasladado al mortuorio para que después sea entregado a la familia. En la asistencia domiciliar o home care el profesional, necesitará llamar al médico para constatar el óbito y si ya hubiera uno dará apoyo a la familia; igualmente ayudará a cuidar del cuerpo, vestirlo y orientará con relación a la obtención del certificado de defunción, los preparativos para el funeral y el traslado del cuerpo para el lugar del velorio o sepultura. Al mismo tiempo es necesario providenciar la retirada del material técnico y equipamientos pertenecientes a la empresa avisando del evento a otras empresas que tengan, alquilado cama de hospital, colchón, silla de ruedas, andador u otros equipamientos especializados, además de separar objetos personales del fallecido para entregarlos a la familia. Esa fase inmediata después de la muerte acostumbra ser de gran tensión y emociones fuertes y el profesional de home care necesita mantener el equilibrio, respeto y orientar en las medidas a tomar y al mismo tiempo se prestará asistencia en la fase de luto familiar, respetando la cultura y los valores. Hay familias que solicitan que los objetos que estaban siendo utilizados por el paciente sean retirados inmediatamente $\mathrm{y}$ otras que no lo permiten durante un tiempo determinado que puede llegar hasta 90 días, alegando que el espíritu del fallecido se desprende a los pocos días. Hay empresas de home care que permiten que el profesional que acompañaba a la paciente en la fase final atienda a la familia inclusive en el velatorio. El Código de Ética de los Profesionales de Enfermería (CEPE) (Conselho Federal de Enfermagem - COFEN - Resolução 311/2007) recuerda el deber de respetar al ser humano en la situación de muerte y post muerte (art. 28), lo que vale tanto para el ambiente del hospital como para el domicilio. Otro punto ético a recordar es la cuestión del sigilo profesional, pues en home care el profesional acaba escuchando conversaciones de disputas de bienes entre potenciales herederos de pacientes en fase terminal o siendo solicitado a ser testigo en decisiones de ultimo momento, como casamiento, testamento, etc. En estas situaciones el profesional debe evitar su participación en cuestiones familiares pues su función es de apoyo asistencial y muy específico.

Entre los factores que justifican el crecimiento de la asistencia domiciliar en el Brasil, Duarte e Diogo (2000) enumeran: los cambios demográficos, con el creciente envejecimiento poblacional; los altos costos del sistema hospitalario, el avance del aparato tecnológico que posibilita la evolución y el prolongamiento del tratamiento de pacientes que sobreviven a múltiples traumas, además de la simplificación de los equipamientos permitiendo la continuidad de la asistencia domiciliar; interés y aceptación de los profesionales, instituciones como la industria farmacéutica y el aumento de la demanda por parte de los pacientes y familiares.

La modalidad de atención domiciliar viene también siendo utilizado por el servicio público, especialmente en el Programa de la Familia - PSF, desde 1991 y fue reglamentado por el Ministerio de la Salud (Portaria n. 1.892) en 1997. Posteriormente, la Portaria n. 2.416, de 1998, consideró que esa modalidad proporcionaría humanización en la atención, además de acompañamiento de pacientes crónicamente dependientes del hospital. Finalmente, la Ley n. 10.424, de 15-01-2002, aumentó un capítulo a la Ley n. 8.080, de 19-091990, y incluye la asistencia domiciliar al Sistema Único de Salud (SUS), creando "en el ámbito del Sistema Único de Salud, la atención e internación a domicilio. En la modalidad de asistencia de atención e ingreso domiciliario se incluyen, los procedimientos médicos, de enfermería, fisioterapéuticos, psicológicos y de asistencia social, entre otros necesarios para el cuidado integral de los pacientes en su domicilio, serán realizados por equipos multidisciplinares que actuarán a nivel de medicina preventiva, terapéutica y rehabilitación y sólo podrán ser realizados por indicación médica, con expresa concordancia del paciente y de su familia".

Considerándose la atención en el domicilio como un modelo de asistencia basado en el sopor- 
te de cuidados al paciente transferido del servicio de hospitalaria para continuar a ser tratado en casa exige un acompañamiento continuo (diario) y a veces, ininterrumpidamente (Duarte, Diogo, 2000 y Cruz, 1994).

Es evidente que en los casos de transferir el paciente del hospital para la casa sea complejo no apenas en la utilización de recursos materiales y equipamientos, sino también en el aspecto humano, transformando el domicilio del paciente en un verdadero hospital virtual. Dada esa alta complejidad, la modalidad de atención domiciliaria es realizada apenas por la red privada según los datos de la Asociación Brasilera de Empresas de Medicina Domiciliar (ABEMID) y el Núcleo Nacional de Empresas de Asistencia Domiciliar (NEAD) ambas son organizaciones de naturaleza empresarial (Conselho Federal de Enfermagem).

No hay que negar que el enfermero que atiende en domicilio ejerce efectivamente una actividad autónoma e independiente, resaltando el carácter liberal de la profesión.

En otra resolución, el COFEN ya dispuso sobre el dimensionamiento del cuadro de profesionales (Conselho Federal de Enfermagem - COFEN Resolução 189, 25-03-1996) de enfermería en las instituciones de salud, estableciendo el cuali-cuantitativo mínimo de los diferentes niveles de formación de los profesionales de enfermería para dar cobertura asistencial mas no incluye bases para el cálculo en asistencia ambulatoria o domiciliar, dejando ese encargo para el buen juicio y el acierto administrativo de los profesionales del área.

Es de destacarse también en lo relacionado a los aspectos ético-profesionales, el enfermero cuenta con el Código de Ética de los Profesionales de Enfermería (Conselho Federal de Enfermagem COFEN- Resolução 311/2007) para garantizar todas las acciones que serán ejecutadas en el domicilio del paciente. Por estar en solitario al tomar decisiones el profesional necesita "evaluar con criterio su competencia técnica y legal, solamente aceptar encargos o atribuciones cuando sea capaz de desempeñar con plena seguridad para sí mismo y para la clientela (CEPE, art. 13).

La supervisión de los cuidadores auxiliares y técnicos de enfermería por los enfermeros es extremadamente importante porque se evaluará el rigor técnico en la ejecución de las actividades de enfermería en los cuidados directos prestados al paciente, ya que es su responsabilidad profesional (Ley $n$. 7.498/86, art 15, y respectivo Decreto reglamentado, art. 13), cuanto menos preparado el cuidador o con poca experiencia el profesional, mayor debe ser el tiempo dedicado en la supervisión directa por el enfermero certificándose de la técnica utilizada hasta los debidos apuntes en la historia clínica domiciliar.

\section{CONSIDERACIONES FINALES}

La expansión de los servicios en todos los países, inclusive en el Brasil, comprueba que ese modelo de atención que era apenas una tendencia mundial ya es una realidad que necesitó de una reglamentación nacional propia (2006), definiendo los servicios, sus condiciones y requisitos para el funcionamiento así como los profesionales que pueden prestar esa modalidad de asistencia y los cuidadores formales e informales.

Para los enfermeros se observa que los países europeos estipulan ciertas exigencias específicas para desenvolver su trabajo en home care, como experiencia profesional previa en actividad de hospital y formación especializada en salud pública y/o enfermería obstétrica, lo que es comprensible considerando las responsabilidades técnicas y legales que irán asumir pues el profesional estará sólo en el domicilio del paciente, no teniendo con quien discutir la mejor decisión a tomar. Es un desafío a enfrentar mas, por otro lado, contamos con una Resolución que no trae antiguas supersticiones terminológicas con relación a la palabra prescripción, y avanzando en el campo de las anotaciones de enfermería en comparación con la legislación del ejercicio profesional, mas siendo necesario mantener alerta para que no ocurran limitaciones de capacidad legal o restricción en las actividades que ya vienen siendo ejercidas por los profesionales de enfermería.

\section{REFERENCIAS BIBLIOGRÁFICAS}

- Brasil. Ministério da Saúde. Resolução da Diretoria Colegiada da Agência Nacional de Vigilância Sanitária - Anvisa RDC n. 11, de 26-01-2006. Dispõe sobre o regulamento técnico de funcionamento de serviços que prestam atenção domiciliar. www.e-legis.anvisa.gov.br 16-02-2006.

- Brasil. Decreto n. 94.406, de 8 de junho de 1987. Regulamenta a Lei n. 7498/86 que dispõe sobre o exercício da 
enfermagem e dá outras providencias. Diário Oficial. Brasília, 09-06-1987. Seção 1, p. 1,fls 8853-8855.

- Brasil. Ministério da Saúde. Portaria n. 1892, de 18-12-1997. Regulamenta o programa de saúde da família. Diário Oficial. Brasília, 20-12-1997.

- Brasil. Ministério da Saúde. Portaria n. 2416, de 23-03-1998. Estabelece requisitos para credenciamento de hospitais e critérios para realização de internação domiciliar no SUS. Diário Oficial. Brasília. 26-031998. p. 106.

- Brasil. Lei n. 10.424, de 15-04-2002. Acrescentou o capítulo VI, sobre subsistema de atendimento e internação domiciliar à Lei n. 8080, de 19-09-1990, que dispõe sobre as condições para a promoção, proteção e recuperação da saúde, reorganização e funcionamento dos serviços correspondentes e dá outras providências (Sistema Único de Saúde). Diário oficial. Brasília. 16 de abril de 2002. Ministério da Saúde. Portaria n. 1892, de 18-12-1997.

- Brasil. Lei n. 7.498, de 25-06-1986. Dispõe sobre a regulamentação do exercício da enfermagem e dá outras providências. In: COREN-SP - Documentos básicos de enfermagem. São Paulo. p. 36-41.

- Carvalho AC. (1965). Histórico da Escola de Enfermagem Lauriston Job Lane. Rev Bras Enfer, Rio de Janeiro, 18 (23):151-6.

- Conselho Federal de Enfermagem - COFEN - Resolução 311/2007. Aprova o Código de Ética dos profissionais de Enfermagem e dá outras providências. In: COREN-SP. Documentos básicos de enfermagem, 2007.

- Conselho Federal de Enfermagem - COFEN - Resolução 267/2001 - aprova atividades de enfermagem em domicilio home care. www.portalcofen.org.br, 22-10-2006.

- Conselho Federal de Enfermagem - COFEN - Resolução 270, de 18-04-2002. Aprova a regulamentação de empresas que prestam serviços de enfermagem domiciliar - home care. www. portalcofen. org.br, 22-02-2006.

- Conselho Federal de Enfermagem - COFEN - Resolução 189, 25-03-1996. Estabelece parâmetros para dimensionamento do quadro de profissionais de enfermagem nas instituições de saúde. In: COFEN- SP - Documentos básicos de enfermagem. São Paulo. p. 144-51 .

- Cruz LP. (1994) Assistência domiciliar: um estudo sobre a formação de profissionais e a prestação de serviços no Estado de São Paulo. Dissertação, São Paulo (SP): Fundação Getulio Vargas.

- Dal Bem LW. (2005) Dimensionamento do pessoal de enfermagem em assistência domiciliária: percepção de gerentes e enfermeiras. Tese (doutorado). Escola de Enfermagem. Universidade de São Paulo, 169p.

- Davoy L. (1997) Structure and services of home care in Norway. International Seminar on Home Care, Budapest, 2526: 65-68.

- Duarte YAO, Diogo MJD. (2000) Atendimento domiciliário: um enfoque gerontológico. São Paulo. Atheneu.

- Ehrenfeld M. (1997) Nursing and home care in Europe. Internacional Seminar on Home Care, Budapest, 25-26:52-57. - Fondations des services d'aide at de soins à diomicile. (2004) Manuel: aide at soins à dinucuke - de vous à nous. Genèver, service de'information-communication.

- Hirschfeld MJ. y Oguisso T. (2002) Visão panorâmica da saúde no mundo e a inserção do home care. Rev Bras Enfer, 55(4):452-9.

- Lopes AD. (2006) Empresas já atendem 30 mil pacientes em casa por mês. Jornal O Estado de São Paulo, edição de 14 de fevereiro, p. 14, Caderno A.

- Madden, PJ. (1997) Home nursing: caring and sharing. International Seminar on Home Care, Budapest, 25-26: 58-60.

- Mucha K. (1997) Home Care and training of home care nurses in Hungary. International Seminar on Home Care, Budapest, 25-26: 69-72.

- World HealthOrganization - Home-based and long-term care. Home care issues at the approach of the 21 th Century from a World Health Organization perspective. A literature review. WHO/HSC/LTH/99.1, Geneva, 1999.

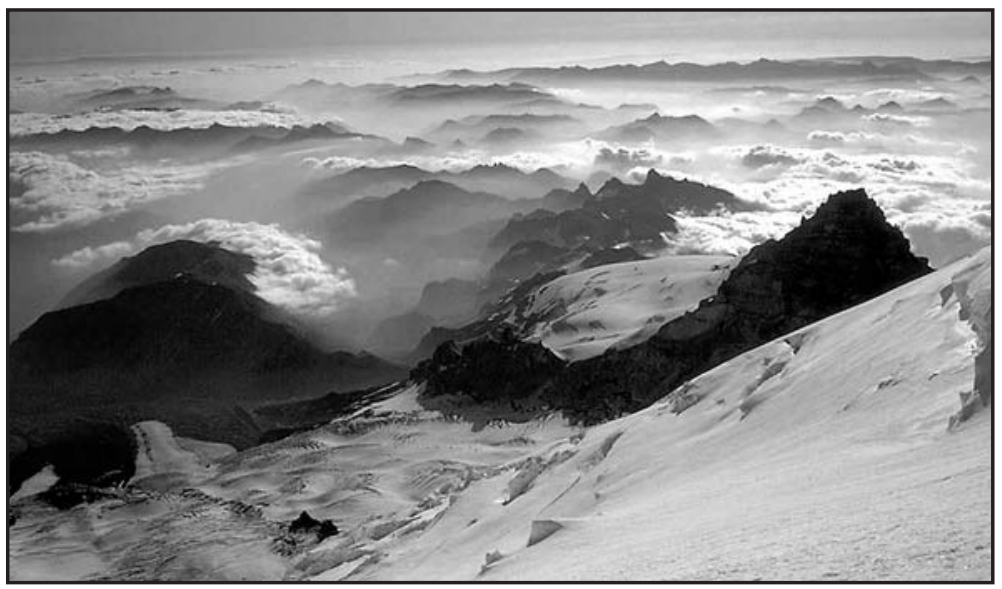

